\title{
There is life in the old horse yet or what else we can learn studying spin- $\frac{1}{2} X Y$ chains
}

\author{
O. Derzhko ${ }^{1,2}$
}

1 Institute for Condensed Matter Physics of the National Academy of Sciences of Ukraine, 1 Svientsitskii Str., 79011 Lviv, Ukraine

2 Chair of Theoretical Physics, Ivan Franko National University of Lviv, 12 Drahomanov Street, 79005 Lviv, Ukraine

Received September 2, 2002

We review some recent results on statistical mechanics of the one-dimensional spin- $\frac{1}{2} X Y$ systems paying special attention to the dynamic and thermodynamic properties of the models with Dzyaloshinskii-Moriya interaction, correlated disorder, and regularly alternating Hamiltonian parameters.

Key words: spin- $\frac{1}{2} X Y$ chains, Dzyaloshinskii-Moriya interaction, correlated disorder, magnetization plateaus, spin-Peierls instability

PACS: $75.10 .-b$

\section{Introductory remarks}

One-dimensional spin- $\frac{1}{2} X Y$ model in a transverse field defined by the Hamiltonian

$$
H=\sum_{n} \Omega s_{n}^{z}+\sum_{n}\left(J^{x} s_{n}^{x} s_{n+1}^{x}+J^{y} s_{n}^{y} s_{n+1}^{y}\right)
$$

is known as the simplest quantum many-body system for which many statistical mechanical calculations can be performed exactly, i.e., without making any simplifying approximations. For more than forty years this model has been a standard testingground for checking various conjectures or new calculation schemes and approaches in statistical mechanics and condensed matter physics. The aim of the present paper is to elucidate some recent results derived for spin- $\frac{1}{2} X Y$ chains and to foresee some further problems which are attractive to study. The interest in spin- $\frac{1}{2} X Y$ chains may be enforced nowadays because of the progress in material sciences (see, for ex- 
ample, the recent report on $\mathrm{Cs}_{2} \mathrm{CoCl}_{4}$, the compound which is a good realization of the famous spin- $\frac{1}{2}$ isotropic $X Y$ chain [1]).

\section{Dynamic properties in fermionic picture}

Spin- $\frac{1}{2} X Y$ chains contain a hidden symmetry which was discovered by applying the Jordan-Wigner transformation: the system of interacting spins (1) can be described in terms of noninteracting spinless fermions (E.Lieb, T.Schultz, D.Mattis). As a result many (although by no means all) statistical mechanical calculations can be performed rigorously. As an example of notorious problems in the statistical mechanics of spin- $\frac{1}{2} X Y$ chains we may mention the analysis of the timedependent correlation functions of $x(y)$ spin components $\left\langle s_{n}^{x}(t) s_{n+m}^{x}\right\rangle, s_{n}^{x}(t)=$ $\exp (\mathrm{i} H t) s_{n}^{x} \exp (-\mathrm{i} H t),\langle(\ldots)\rangle=\operatorname{Tr}(\exp (-\beta H)(\ldots)) / \operatorname{Tr}(\exp (-\beta H))$. Since the relation between the $x$ spin component attached to a certain site and the on-site creation and annihilation operators of fermions is nonlocal and involves the occupationnumber operators of fermions at all previous sites, the problem of applying the Wick-Bloch-de Dominicis theorem to a product of a huge number of multipliers arises. The result can be written compactly as the Pfaffian of an antisymmetric matrix (generally speaking of huge sizes) constructed from the elementary contractions and hence a further analytical analysis becomes not simple. The problem has been solved to some extent by elaborating the numerical schemes for computation

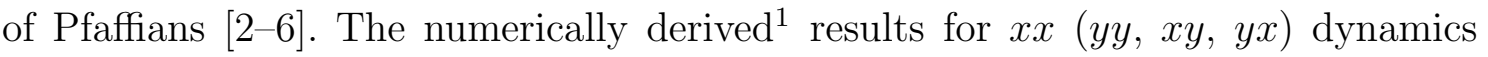
supplemented by the analytical results for $z z$ dynamics [8] permit to work out the theory of dynamic properties of spin- $\frac{1}{2} X Y$ chains in the fermionic picture (G.Müller with coworkers) and thus to explain the peculiarities of responses of the spin system to small external perturbations $[8,9]$.

Let us sketch briefly the linear response theory of the spin chain in the fermionic language considering for simplicity the isotropic $X Y$ model $\left(J^{x}=J^{y}=J\right)$. The $z z$ dynamic structure factor for this model which is given by

$$
\begin{array}{r}
S_{z z}(\kappa, \omega)=\int_{-\pi}^{\pi} \mathrm{d} \kappa_{1} n_{\kappa_{1}}\left(1-n_{\kappa_{1}-\kappa}\right) \delta\left(\omega+\Lambda_{\kappa_{1}}-\Lambda_{\kappa_{1}-\kappa}\right), \\
\Lambda_{\kappa}=\Omega+J \cos \kappa, \quad n_{\kappa}=\frac{1}{1+\exp \left(\beta \Lambda_{\kappa}\right)}
\end{array}
$$

suggests the following interpretation $[8,10]$. Consider at first the high-temperature limit $\beta \rightarrow 0$ when $n_{\kappa} \rightarrow \frac{1}{2}$ and hence $S_{z z}(\kappa, \omega)(2)$ becomes independent of $\Omega$. Applying the infinitesimally small external field (directed along $z$ axis) characterized by the wave vector $\kappa$ and frequency $\omega$ we observe that the responsive magnetization (directed along $z$ axis) is determined by generation of the two fermions with energies $\Lambda_{\kappa_{1}}$ and $\Lambda_{\kappa_{2}}$ under the restrictions $\kappa=\kappa_{1}-\kappa_{2}$ and $\omega=-\Lambda_{\kappa_{1}}+\Lambda_{\kappa_{2}}=$

\footnotetext{
${ }^{1}$ The numerical approach is not restricted to the uniform chains and can be easily applied to the nonuniform chains in which the Hamiltonian parameters vary regularly along the chain with a finite period or are random variables with a given probability distribution [7].
} 
$a$
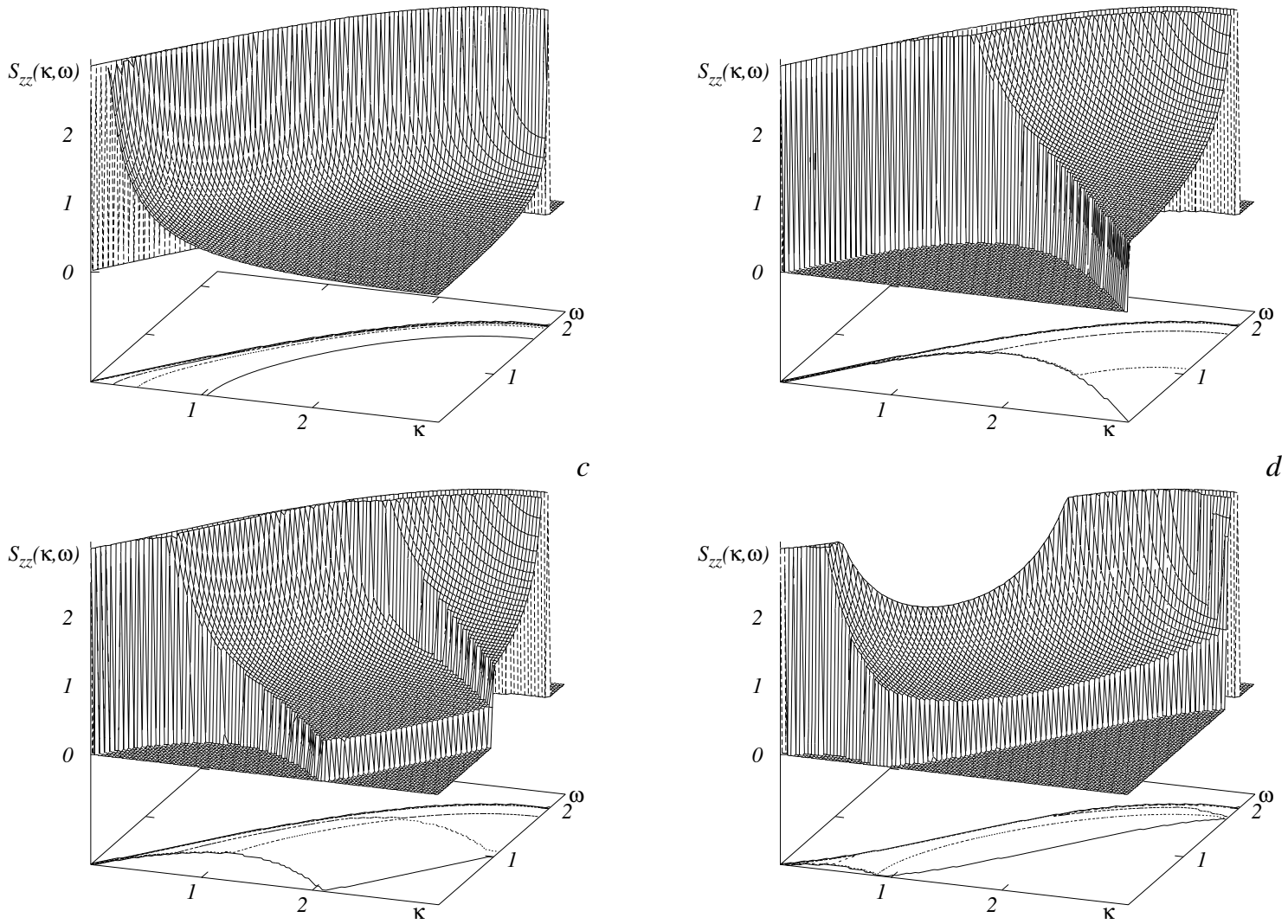

Figure 1. $S_{z z}(\kappa, \omega)$ for the isotropic $X Y$ chain in a transverse field $(J=1)$ at infinite temperature $\beta=0$ (a) and zero temperature $\beta \rightarrow \infty$ (b: $\Omega=0$, c: $\Omega=0.5, \mathrm{~d}: \Omega=0.9)$.

$2 J \sin \frac{\kappa}{2} \sin \left(\kappa_{1}-\frac{\kappa}{2}\right)$. The "dummy" wave vector $\kappa_{1}$ in $(2)$ varies within the region $-\pi \leqslant \kappa_{1} \leqslant \pi$. As a result, such an experimental probe "measures" a continuum of the two-fermion excitations in the $\kappa-\omega$ plane. The upper boundary of the twofermion continuum is given by $\omega=2\left|J \sin \frac{\kappa}{2}\right|$ and $S_{z z}(\kappa, \omega)$ exhibits divergence along this line as it follows from (2). At low temperatures $\beta \rightarrow \infty$, the Fermi factors in (2) come into play and $S_{z z}(\kappa, \omega)$ becomes dependent on $\Omega$. The additional conditions $\Lambda_{\kappa_{1}}<0$ and $\Lambda_{\kappa_{2}}>0$ lead to the appearance of the lower boundary (for example, $\omega=|J \sin \kappa|$ if $\Omega=0)$ at which a finite value of $S_{z z}(\kappa, \omega)$ jumps to zero. The transverse field (which plays a role of the chemical potential in the fermionic picture) effects the lower boundary of the two-fermion continuum in the $\kappa-\omega$ plane and the redistribution of the values of $S_{z z}(\kappa, \omega)$ in the $\kappa$ - $\omega$ plane at low temperatures. For $|\Omega|>|J| S_{z z}(\kappa, \omega)$ vanishes everywhere in the $\kappa$ - $\omega$ plane. The above-said can be seen in figure 1 where some typical results illustrating $z z$ dynamics are reported.

Contrary to the $z z$ dynamics the $x x$ dynamics is more involved: we do not know the explicit expression for $S_{x x}(\kappa, \omega)$ similar to (2). Equation (2) arises after computation of the average of the product of four Fermi operators

$$
\left\langle\left(1-2 c_{n}^{+}(t) c_{n}(t)\right)\left(1-2 c_{n+m}^{+} c_{n+m}\right)\right\rangle
$$


that is obviously the two-fermion quantity. The $x x$ dynamic structure factor contains the averages like

$$
\begin{array}{r}
\left\langle\left(1-2 c_{1}^{+}(t) c_{1}(t)\right) \ldots\left(1-2 c_{n-1}^{+}(t) c_{n-1}(t)\right)\left(c_{n}^{+}(t)+c_{n}(t)\right)\right. \\
\left.\quad \times\left(1-2 c_{1}^{+} c_{1}\right) \ldots\left(1-2 c_{n+m-1}^{+} c_{n+m-1}\right)\left(c_{n+m}^{+}+c_{n+m}\right)\right\rangle
\end{array}
$$

and thus it is a many-fermion quantity. However, the numerical calculations show that the two-fermion continuum dominates the low-temperature behaviour of the $x x$ dynamic structure factor. Although $S_{x x}(\kappa, \omega)$ is not restricted to the two-fermion continuum region in the $\kappa$ - $\omega$ plane and has nonzero value above the upper boundary of the two-fermion continuum (demonstrating the effects of the many-fermion continua [11]) its value outside the two-fermion continuum is rather small. $S_{x x}(\kappa, \omega)$ may be described by several washed-out excitation branches following roughly the two-fermion continuum boundaries. Further studies are required to clarify why twofermion features rule the many-fermion quantity $S_{x x}(\kappa, \omega)$. For $|\Omega|>|J|$ the zerotemperature $x x$ dynamic structure factor $S_{x x}(\kappa, \omega)$ shows a single $\delta$-peak along the fermion branch $\omega=\Lambda_{\kappa}$. In the high-temperature limit the low-temperature structures in $\kappa-\omega$ plane disappear and $S_{x x}(\kappa, \omega)$ becomes $\kappa$-independent in agreement with exact calculations for $\beta=0$ [12]. Alternatively the $x x$ dynamics can be examined using a bosonization treatment [13], however, such an analysis is restricted to low-energy physics and only a small region in the $\kappa-\omega$ plane can be explored by this approach.

The described analysis of the dynamic properties may be extended to the anisotropic $X Y$ interaction $\left(J^{x} \neq J^{y}\right)[8]$ and the dimerized isotropic $X Y$ interaction $\left(J^{x}=J^{y} \rightarrow J\left(1-(-1)^{n} \delta\right), 0 \leqslant \delta \leqslant 1\right.$ is the dimerization parameter) [8,9]. Apparently, the exhaustive study of the $z z$ dynamics for the former case [8] should be still supplemented by the corresponding analysis of the $x x$ dynamics whereas the case when both anisotropy and dimerization are present requires a separate study. Recently, the effects of periodic inhomogeneity on the dynamic susceptibility $\chi_{z z}(\kappa, \omega)$ (but not $\chi_{x x}(\kappa, \omega)$ ) have been reported in the paper on dynamics of isotropic $X Y$ model on one-dimensional superlattices [14]. The $x x$ dynamic quantities for the case of extremely anisotropic $X Y$ interaction $\left(J^{y}=0\right)$, i.e., for the spin- $\frac{1}{2}$ transverse Ising chain, are of interest for interpreting the experimental data on the dynamic dielectric permittivity of the quasi-one-dimensional hydrogen-bonded ferroelectric compound $\mathrm{CsH}_{2} \mathrm{PO}_{4}[15,16]$.

To end up this section, let us note that the dynamic properties of two-dimensional quantum spin models can be also explained in terms of the two-fermion continuum, however, such a picture may have only an approximate meaning [17]. Another interesting question is to contrast the results for dynamic structure factors of spin- $\frac{1}{2}$ and spin-1 chains [18].

\section{Dzyaloshinskii-Moriya interaction}

The Dzyaloshinskii-Moriya interaction is often present in the low-dimensional quantum magnets (see, for example, a recent paper [19]). It is generally known 
that the Dzyaloshinskii-Moriya interaction $\sum_{n} \mathbf{D} \cdot\left(\mathbf{s}_{n} \times \mathbf{s}_{n+1}\right)$ being added to the Hamiltonian (1) does not destroy the rigorous treatment if $\mathbf{D}=(0,0, D)[20]^{2}$.

The main effect of the Dzyaloshinskii-Moriya interaction is the loss of the symmetry of elementary excitation energies with respect to the change $\kappa \rightarrow-\kappa$ :

$$
\Lambda_{\kappa}=D \sin \kappa+\sqrt{\left(\Omega+\frac{J^{x}+J^{y}}{2} \cos \kappa\right)^{2}+\left(\frac{J^{x}-J^{y}}{2}\right)^{2} \sin ^{2} \kappa \neq \Lambda_{-\kappa}}
$$

$[20,22]$. In the presence of the Dzyaloshinskii-Moriya interaction some remarkable changes in the thermodynamic and dynamic properties of spin chains may occur. Consider, for example, the isotropic $X Y$ chain. Since the Dzyaloshinskii-Moriya interaction for such a chain (even inhomogeneous one) can be eliminated by the local rotations in spin space around the $z$ axis

$$
\begin{aligned}
s_{n}^{x} \cos \phi_{n}+s_{n}^{y} \sin \phi_{n} & \rightarrow s_{n}^{x}, \quad-s_{n}^{x} \sin \phi_{n}+s_{n}^{y} \cos \phi_{n} \\
\phi_{n} & =\varphi_{1}+\ldots+s_{n-1}^{y}, \quad \tan \varphi_{m}=\frac{D_{m}}{J_{m}}
\end{aligned}
$$

resulting in a model with isotropic $X Y$ interaction $\sqrt{J_{n}^{2}+D_{n}^{2}}$ the $z z$ dynamics remains as for a chain without Dzyaloshinskii-Moriya interaction, however, with renormalized energy scale $J \rightarrow \sqrt{J^{2}+D^{2}}$. In contrast, the $x x$ dynamic quantities according to (3) involve

$$
\begin{aligned}
\left\langle s_{n}^{x}(t) s_{n+m}^{x}\right\rangle_{J, D}= & \quad \cos \phi_{n} \cos \phi_{n+m}\left\langle s_{n}^{x}(t) s_{n+m}^{x}\right\rangle_{\sqrt{J^{2}+D^{2}}, 0} \\
- & \cos \phi_{n} \sin \phi_{n+m}\left\langle s_{n}^{x}(t) s_{n+m}^{y}\right\rangle_{\sqrt{J^{2}+D^{2}}, 0} \\
- & \sin \phi_{n} \cos \phi_{n+m}\left\langle s_{n}^{y}(t) s_{n+m}^{x}\right\rangle_{\sqrt{J^{2}+D^{2}}, 0} \\
+ & \sin \phi_{n} \sin \phi_{n+m}\left\langle s_{n}^{y}(t) s_{n+m}^{y}\right\rangle_{\sqrt{J^{2}+D^{2}}, 0}
\end{aligned}
$$

(for the homogeneous chain $\left.\phi_{n}=(n-1) \varphi, \tan \varphi=D / J\right)$. In view of (4) the relation between the $z z$ and $x x$ dynamics discussed in the previous section $\left(S_{x x}(\kappa, \omega)\right.$ at low temperatures exhibits the washed-out excitation branches which roughly follow the boundaries of the two-fermion continuum which determines $S_{z z}(\kappa, \omega)$ ) may appear to be more intricate [23]. In the next sections 4 and 5 we give further examples of how the Dzyaloshinskii-Moriya interaction manifests itself in the properties of spin- $\frac{1}{2}$ $X Y$ chains.

\section{Correlated off-diagonal and diagonal disorder}

The Jordan-Wigner transformation maps the spin- $\frac{1}{2}$ isotropic $X Y$ chain in a transverse field onto the chain of tight-binding spinless fermions with the on-site

\footnotetext{
${ }^{2}$ Let us note that in some cases the Dzyaloshinskii-Moriya interaction can be eliminated by the corresponding spin-coordinate transformation [21] (see, for example, equation (3)).

${ }^{3}$ This is not the case if $X Y$ interaction is anisotropic; the $z z$ dynamics of the model with extremely anisotropic $X Y$ interaction, i.e., the transverse Ising chain with Dzyaloshinskii-Moriya interaction, was considered in [22].
} 
energy $\Omega$ and hopping $I=J / 2$. If the transverse fields are independent random variables (diagonal disorder) each with the Lorentzian probability distribution

$$
p\left(\Omega_{n}\right)=\frac{1}{\pi} \frac{\Gamma}{\left(\Omega_{n}-\Omega_{0}\right)^{2}+\Gamma^{2}}
$$

the resulting fermionic model is the one-dimensional version of the Lloyd model. The density of states,

$$
\overline{\rho(E)}=\overline{\frac{1}{N} \sum_{k=1}^{N} \delta\left(E-\Lambda_{k}\right)}, \quad \overline{(\ldots)}=\ldots \int \mathrm{d} \Omega_{n} p\left(\Omega_{n}\right) \ldots(\ldots),
$$

for the Lloyd model can be found exactly [24]. Going far beyond the idea of H.Nishimori we may consider a spin model with the correlated off-diagonal and diagonal Lorentzian disorder which after fermionization reduces to the one-dimensional version of the extended Lloyd model introduced by W.John and J.Schreiber. Namely, we consider the isotropic $X Y$ model with independent random exchange interactions (off-diagonal disorder) given by the Lorentzian probability distribution

$$
p\left(\ldots, J_{n}, \ldots\right)=\prod_{n} p\left(J_{n}\right)=\prod_{n} \frac{1}{\pi} \frac{\Gamma}{\left(J_{n}-J_{0}\right)^{2}+\Gamma^{2}} .
$$

Moreover, we consider the correlated off-diagonal and diagonal disorder assuming that the on-site transverse fields in the chain are determined by the surrounding exchange interactions according to the relation

$$
\Omega_{n}-\Omega_{0}=\frac{a}{2}\left(J_{n-1}+J_{n}-2 J_{0}\right), \quad a \text { is real, } \quad|a| \geqslant 1 .
$$

Then the density of states $\overline{\rho(E)}$ yielding the thermodynamic quantities of the introduced random spin chain can be calculated exactly $[25,26]$. To get $\overline{\rho(E)}$ we must calculate the diagonal Green functions $\overline{G_{n n}^{\mp}(E)}$, since

$$
\overline{\rho(E)}=\mp \frac{1}{\pi} \Im \overline{G_{n n}^{\mp}(E)}
$$

The set of equations of motion for $G_{n m}^{\mp}(E \pm \mathrm{i} \epsilon), \epsilon \rightarrow+0$ can be averaged using contour integration in complex planes of random (Lorentzian) variables $J_{n}$. Using the Gershgorin criterion we find the set of equations for the averaged Green functions which has the same structure as before averaging but possesses translational symmetry. As a result we obtain the desired quantities $\overline{G_{n m}^{\mp}(E)}$ and hence all thermodynamic quantities.

In figures $2 \mathrm{a}$ and $2 \mathrm{~b}$ we present $\overline{\rho(E)}$ in the most interesting region $|a| \rightarrow 1$, when $\overline{\rho(E)}$ becomes not symmetric with respect to the change $E-\Omega_{0} \rightarrow-\left(E-\Omega_{0}\right)$. Such asymmetry immediately yields a nonzero (average) transverse magnetization

$$
\overline{m_{z}}=-\frac{1}{2} \int \mathrm{d} E \overline{\rho(E)} \tanh \frac{\beta E}{2} \neq 0
$$



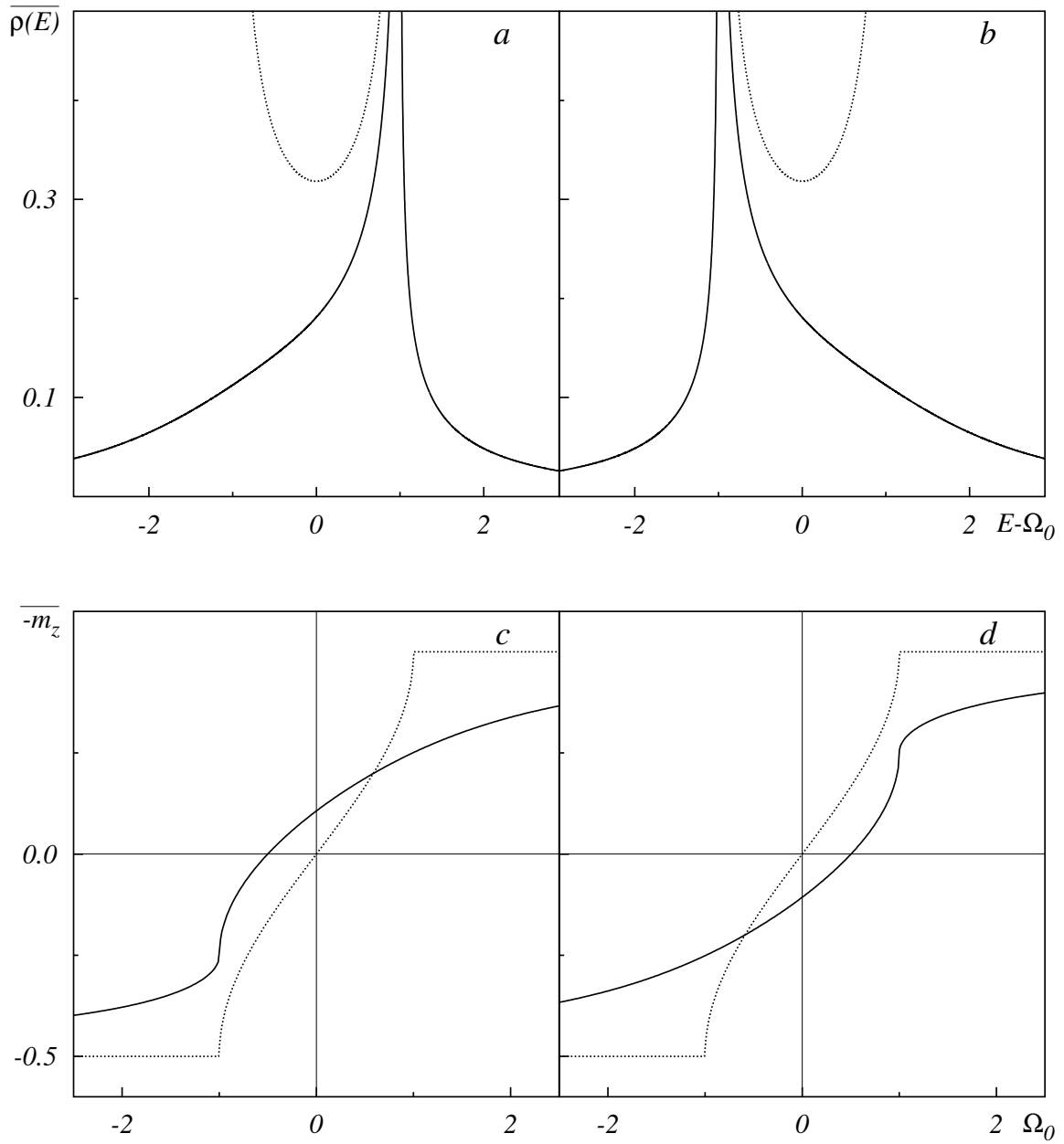

Figure 2. The density of states $(\mathrm{a}, \mathrm{b})$ and the transverse magnetization $(\mathrm{c}, \mathrm{d})$ of the isotropic $X Y$ chain with correlated Lorentzian disorder $\left(J_{0}=1, \Gamma=1\right.$, $a=-1(\mathrm{a}, \mathrm{c}), a=1(\mathrm{~b}, \mathrm{~d}))$. The dotted curves correspond to the nonrandom case $(\Gamma=0)$.

at low temperatures $\beta \rightarrow \infty$ at zero (average) transverse field $\Omega_{0}=0$ (figures $2 \mathrm{c}$ and $2 \mathrm{~d}$ ). Let us consider more closely this somewhat unexpected magnetic property of the introduced random spin chain. For a certain random realization of the chain defined by (5), (6) one may expect the same numbers of sites surrounded by stronger than $J_{0}$ exchange interactions as the sites surrounded by weaker than $J_{0}$ exchange interactions. Because of (6) for $\Omega_{0}=0$ the transverse fields at the former and at the latter sites have the same value but the opposite signs giving as a result $\sum_{n} \Omega_{n}=0$. On the other hand, one may expect that the sites surrounded by strong isotropic $X Y$ exchange interactions exhibit small $z$ magnetization whereas the sites surrounded by weak isotropic $X Y$ exchange interactions exhibit large $z$ magnetization (in the opposite direction). As a result, the average transverse magnetization has a nonzero value. As $|a|$ increases, a difference in the oppositely directed $z$ magnetizations becomes smaller. Thus, a nonzero $\overline{m_{z}}$ at $\Omega_{0}=0$ appears owing to the imposed relation (6) which expresses the condition of correlated disorder. 
Some further insight into the origin of the asymmetry of $\overline{\rho(E)}$ can be obtained after examining the moments of the density of states

$$
\begin{aligned}
M^{(r)} & \equiv \int \mathrm{d} E E^{r} \rho(E) \\
& =\frac{1}{N} \sum_{n=1}^{N}\left\langle\left\{\left[\ldots\left[c_{n}, H\right], \ldots, H\right], c_{n}^{+}\right\}\right\rangle
\end{aligned}
$$

(here $H$ is the Hamiltonian of fermions which represent the spin chain). It is just the correlated disorder that yields a nonzero third moment $\overline{M^{(3)}} \neq 0^{4}$ at $\Omega_{0}=0$. For not correlated off-diagonal and diagonal disorders one gets $\overline{M^{(3)}}=0$ at $\Omega_{0}=0$. The moments of the density of states can be calculated for any probability distribution of random variables $p\left(J_{n}\right)$ (not necessarily for the Lorentzian probability distribution), for example, for the rectangle probability distribution. These calculations explicitly demonstrate the cause of the asymmetry appearance in $\overline{\rho(E)}[27]$. Some other results on the effects of correlated disorder can be found in $[10,28,29]$.

Finally, let us remark that the considered spin model with correlated Lorentzian disorder may be extended by introducing the nonrandom Dzyaloshinskii-Moriya interaction $D$. Another extension is to assume the exchange interaction to be nonrandom $J_{n}=J$ whereas the Dzyaloshinskii-Moriya interactions $D_{n}$ to be independent random Lorentzian variables determining the transverse fields according to (6). Both models are related to each other through a certain sequence of rotations of spin axes around the $z$ axis (being applied to the Hamiltonian with the exchange interactions $D_{n}$ and the Dzyaloshinskii-Moriya interactions $-J_{n}$ it gives the Hamiltonian with the exchange interactions $J_{n}$ and the Dzyaloshinskii-Moriya interactions $D_{n}$ ) and hence it is sufficient to consider only one of them. Considering, for example, the former model one finds that the nonrandom Dzyaloshinskii-Moriya interaction may lead to the recovery of the symmetry with respect to the change $E-\Omega_{0} \rightarrow-\left(E-\Omega_{0}\right)$ and hence to a decrease of the nonzero value of $\overline{m_{z}}$ at $\Omega_{0}=0$. Such an effect becomes also apparent after calculating the moments of the density of states $\overline{M^{(2)}}$ and $\overline{M^{(3)}}$ (7) for this model.

To end up this section, let us note that a different random spin- $\frac{1}{2} X Y$ chains were rigorously analytically examined by Th.M.Nieuwenhuizen and coauthors [30].

\section{Effects of regularly alternating bonds and fields}

\subsection{Continued fractions}

The quantum spin chains with regularly alternating Hamiltonian parameters can model dimerized ( $l$-merized) chains, one-dimensional superlattices, one-dimensional

\footnotetext{
${ }^{4}$ Here $\overline{(\ldots)}$ denotes the random averaging either for the correlated off-diagonal and diagonal disorder or for the independent off-diagonal (with the probability distribution $p\left(J_{n}\right)$ ) and diagonal (with the probability distribution $p\left(\Omega_{n}\right)=\int \mathrm{d} J_{n-1} \int \mathrm{d} J_{n} p\left(J_{n-1}\right) p\left(J_{n}\right) \delta\left(\Omega_{n}-\Omega_{0}-\frac{a}{2}\left(J_{n-1}+J_{n}\right.\right.$ $\left.\left.-2 J_{0}\right)\right)$ ) disorders.
} 
decorated chains etc. The case of spin- $\frac{1}{2} X Y$ chains supplemented by the continued fraction approach (for other approaches see [31]) remains amenable for rigorous analysis of the thermodynamic properties if the exchange interaction is isotropic $\left(J_{n}^{x}=J_{n}^{y}\right)$ [32], extremely anisotropic $\left(J_{n}^{y}=0\right)$ [33] or if $\Omega_{n}=0$ [34]. The thermodynamic quantities of the regularly alternating isotropic $X Y$ chain in a transverse field can be obtained through the density of states $\rho(E)=\frac{1}{N} \sum_{k=1}^{N} \delta\left(E-\Lambda_{k}\right)$. The thermodynamic quantities of the regularly alternating transverse Ising chain and the regularly alternating anisotropic $X Y$ chain without transverse field can be obtained through the density of states $R\left(E^{2}\right)=\frac{1}{N} \sum_{k=1}^{N} \delta\left(E^{2}-\Lambda_{k}^{2}\right)$.

Let us recall that after fermionization of the isotropic $X Y$ chain in a transverse field $\left(J_{n}^{x}=J_{n}^{y}=2 I_{n}\right)$ one faces the Hamiltonian which is a bilinear fermion form. While making it diagonal one arrives at the set of equations

$$
I_{n-1} g_{k, n-1}+\left(\Omega_{n}-\Lambda_{k}\right) g_{k n}+I_{n} g_{k, n+1}=0,
$$

here $g_{k n}$ are the coefficients of the linear transformation which make the initial bilinear fermion form diagonal and $\Lambda_{k}$ are the resulting elementary excitation energies. Therefore, introducing the Green functions $\mathcal{G}_{n m}(E)$ according to

$$
-I_{n-1} \mathcal{G}_{n-1, m}(E)+\left(E-\Omega_{n}\right) \mathcal{G}_{n m}(E)-I_{n} \mathcal{G}_{n+1, m}(E)=\delta_{n m}
$$

one gets the density of states

$$
\rho(E)=\mp \frac{1}{\pi N} \sum_{n=1}^{N} \Im \mathcal{G}_{n n}(E \pm \mathrm{i} \epsilon), \quad \epsilon \rightarrow+0 .
$$

For the transverse Ising chain $\left(J_{n}^{x}=2 I_{n}, J_{n}^{y}=0\right)$ instead of (8) and (9) we have

$$
\Omega_{n-1} I_{n-1} \Phi_{k, n-1}+\left(\Omega_{n}^{2}+I_{n-1}^{2}-\Lambda_{k}^{2}\right) \Phi_{k n}+\Omega_{n} I_{n} \Phi_{k, n+1}=0
$$

and

$$
-\Omega_{n-1} I_{n-1} \mathcal{G}_{n-1, m}\left(E^{2}\right)+\left(E^{2}-I_{n-1}^{2}-\Omega_{n}^{2}\right) \mathcal{G}_{n m}\left(E^{2}\right)-\Omega_{n} I_{n} \mathcal{G}_{n+1, m}\left(E^{2}\right)=\delta_{n m},
$$

respectively, whereas for the anisotropic $X Y$ chain without field $\left(J_{n}^{x}=2 I_{n}^{x}, J_{n}^{y}=2 I_{n}^{y}\right.$, $\Omega_{n}=0$ ) instead of (8) and (9) we have

$$
I_{n-2}^{y} I_{n-1}^{x} \Phi_{k, n-2}+\left(I_{n-1}^{x}{ }^{2}+I_{n}^{y 2}-\Lambda_{k}^{2}\right) \Phi_{k n}+I_{n}^{y} I_{n+1}^{x} \Phi_{k, n+2}=0
$$

and

$$
-I_{n-2}^{y} I_{n-1}^{x} \mathcal{G}_{n-2, m}\left(E^{2}\right)+\left(E^{2}-I_{n-1}^{x}{ }^{2}-I_{n}^{y 2}\right) \mathcal{G}_{n m}\left(E^{2}\right)-I_{n}^{y} I_{n+1}^{x} \mathcal{G}_{n+2, m}\left(E^{2}\right)=\delta_{n m},
$$

respectively. For the last two models the Green functions $\mathcal{G}_{n m}\left(E^{2}\right)$ yield the density of states

$$
R\left(E^{2}\right)=\mp \frac{1}{\pi N} \sum_{n=1}^{N} \Im \mathcal{G}_{n n}\left(E^{2} \pm \mathrm{i} \epsilon\right), \quad \epsilon \rightarrow+0 .
$$


For a general case of the anisotropic $X Y$ chain in a transverse field a set of equations like (10) or (12) is five diagonal banded (but not three diagonal banded as (8), (10) or (12)) and the next step, i.e., the continued fraction representation for the diagonal Green functions is less evident. According to equation (9), (11) or (13) the diagonal Green functions for all these models can be represented in terms of continued fractions. For example, from (13) it immediately follows that

$$
\begin{gathered}
\mathcal{G}_{n n}\left(E^{2}\right)=\frac{1}{E^{2}-I_{n-1}^{x}{ }^{2}-I_{n}^{y 2}-\Delta_{n}^{-}-\Delta_{n}^{+}}, \\
\Delta_{n}^{-}=\frac{I_{n-2}^{y}{ }^{2} I_{n-1}^{x}{ }^{2}}{E^{2}-I_{n-3}^{x}{ }^{2}-I_{n-2}^{y}{ }^{2}-\frac{I_{n-4}^{y}{ }^{2} I_{n-3}^{x}{ }^{2}}{E^{2}-I_{n-5}^{x}{ }^{2}-I_{n-4}^{y}{ }^{2}-} \cdot} \\
\Delta_{n}^{+}=\frac{I_{n}^{y 2} I_{n+1}^{x}{ }^{2}}{E^{2}-I_{n+1}^{x}{ }^{2}-I_{n+2}^{y}{ }^{2}-\frac{I_{n+2}^{y}{ }^{2} I_{n+3}^{x}{ }^{2}}{E^{2}-I_{n+3}^{x}{ }^{2}-I_{n+4}^{y}{ }^{2}-} \cdot}
\end{gathered}
$$

If now the Hamiltonian parameters are periodic with any finite period $p$ the continued fractions $\Delta_{n}^{\mp}$ in (14) become periodic and can be evaluated by solving square equations. As a result, we get the exact expressions for $\mathcal{G}_{n n}\left(E^{2}\right)$ (or $\mathcal{G}_{n n}(E)$ for the isotropic $X Y$ chain) and hence for all thermodynamic quantities of the regularly alternating spin chains in question.

Let us remark, that the thermodynamic quantities of the anisotropic $X Y$ chain in a transverse field of period 2 were obtained in [35] in a different manner on a quite general background. This scheme, however, becomes cumbersome if the period of nonuniformity increases. Further development of a general approach to the study of thermodynamic quantities and spin correlation functions has been reported by L.L.Gonçalves with coworkers in connection with spin- $\frac{1}{2} X Y$ models on onedimensional superlattices.

\subsection{Magnetization processes}

The most spectacular manifestation of the effects of regular alternation can be seen in the magnetization processes at low temperatures. Consider at first the isotropic $X Y$ chain. Owing to regularly alternating parameters of the Hamiltonian the fermion band splits into several subbands the number of which does not exceed the period of regular inhomogeneity. This circumstance immediately suggests that the zero-temperature dependence

$$
m_{z}=-\frac{1}{2} \int \mathrm{d} E \rho(E) \tanh \frac{\beta E}{2}
$$

on $\Omega$ (we have assumed that $\Omega_{n}=\Omega+\Delta \Omega_{n}$ ) consists of horizontal parts (plateaus) which appear when $E=0$ remains in between subbands (given by $\rho(E)$ ) with changing of $\Omega$ separated by the parts of varying $m_{z}$ which appear when $E=0$ remains within subbands with $\Omega$ changing. Clearly, the number of plateaus does 
not exceed the period of nonuniformity. Moreover, their heights $-s \leqslant m_{z} \leqslant s$ are in agreement with the condition: $p\left(s-m_{z}\right)=$ integer with $s=\frac{1}{2}$ [36]. This conjecture was suggested on the model-independent background for a general spin$s$ chain with axial symmetry in a uniform magnetic field using the Lieb-SchultzMattis theorem and the bosonization arguments. Obviously, spin- $\frac{1}{2} X Y$ chain is an easy case (represented by noninteracting fermions) contrary to the chains with Heisenberg exchange interaction or higher spins $s>\frac{1}{2}$. On the other hand, the magnetization curve for spin- $\frac{1}{2} X Y$ chain can be obtained explicitly. Moreover, the elaborated scheme also permits to obtain the local (on-site) magnetizations. The onsite magnetizations exhibit plateaus which begin and end up at the same values of $\Omega$ as for the total magnetization, however, the plateaus heights (i.e., the values of the on-site magnetizations) are not universal quantities no longer obeying the abovementioned condition and strongly depend on a concrete set of the Hamiltonian parameters. Moreover, a sequence of sites $n_{1}, n_{2}, \ldots, n_{p}$ satisfying the inequalities $m_{z n_{1}} \leqslant m_{z n_{2}} \leqslant \ldots \leqslant m_{z n_{p}}$ depends on the value of the applied field $\Omega$. The step-like behaviour of the zero-temperature dependence $m_{z}\left(m_{z n}\right)$ vs. $\Omega$ is accompanied by the corresponding zero-temperature behaviour $\chi_{z}=\partial m_{z} / \partial \Omega\left(\chi_{z_{n}}=\partial m_{z n} / \partial \Omega\right)$ vs. $\Omega$. The zero-temperature dependence $\chi_{z}$ on $\Omega$ behaves like $-\rho(E=0)$ vs. $\Omega$ thus reproducing the density of states $\rho(E)$.

In contrast to the isotropic $X Y$ chain, a regular alternation of the parameters of the transverse Ising chain Hamiltonian does not lead to plateaus in the zerotemperature dependence $m_{z}$ on $\Omega$. The difference arises owing to the anisotropy of $X Y$ interaction: if $J_{n}^{x} \neq J_{n}^{y}$ one finds that $\left[\sum_{n} s_{n}^{z}, H\right] \neq 0$. Thus, even if the spin system remains in the same ground state $|0\rangle$ with varying $\Omega$ the magnetization $\left\langle 0\left|\sum_{n} s_{n}^{z}\right| 0\right\rangle$, nevertheless, varies with varying $\Omega$. As a result the dependence $m_{z}$ vs. $\Omega$ does not exhibit horizontal parts. On the other hand, in the vicinity of some fields $\Omega^{\star}$ (critical fields) the magnetization may behave as $m_{z} \sim\left(\Omega-\Omega^{\star}\right) \ln \left|\Omega-\Omega^{\star}\right|$ and hence the susceptibility as $\chi_{z} \sim \ln \left|\Omega-\Omega^{\star}\right|$. For the uniform chain there are two critical fields $\Omega^{\star}= \pm|I|$. If the bonds become regularly alternating only the values of $\Omega^{\star}$ change. For example, $\Omega^{\star}= \pm \sqrt{\left|I_{1} I_{2}\right|}$ for $p=2, \Omega^{\star}= \pm \sqrt[3]{\left|I_{1} I_{2} I_{3}\right|}$ for $p=3$ etc. If the fields become regularly alternating not only the values of the critical fields vary but the number of the critical fields may change. For example, assuming $\Omega_{1,2}=\Omega \pm \Delta \Omega, \Delta \Omega \geqslant 0$ one has either two critical fields $\Omega^{\star}= \pm \sqrt{\Delta \Omega^{2}+\left|I_{1} I_{2}\right|}$ if $\Delta \Omega<\sqrt{\left|I_{1} I_{2}\right|}$ or four critical fields $\Omega^{\star}= \pm \sqrt{\Delta \Omega^{2} \pm\left|I_{1} I_{2}\right|}$ if $\Delta \Omega>\sqrt{\left|I_{1} I_{2}\right|}$. The transverse field is a controlling parameter of the second-order quantum phase transition in the spin- $\frac{1}{2}$ Ising chain in a transverse field [37]. Thus, a number of the quantum phase transition points governed by $\Omega$ in this model may increase due to a regular alternation of the transverse fields presuming the strength of inhomogeneity is sufficiently strong. For example, for $p=2$ there may be either two or four quantum phase transition points, for $p=3$ there may be either two, or four, or six quantum phase transition points etc. The critical behaviour remains unchanged and is just like for the temperature driven phase transition in the square-lattice Ising model. For example, the zero-temperature dependence $\chi_{z}$ vs. $\Omega$ always exhibits logarithmic singularities the number of which depends on a concrete set of the Hamiltonian 
parameters. It seems interesting to trace the changes in magnetization processes as anisotropy of the exchange interaction varies between the isotropic and extremely anisotropic limits.

It is instructive to compare the zerotemperature magnetization processes in the quantum and classical $X Y$ chains $^{5}$. A classical chain consists of arrows (vectors) $\mathbf{s}=(s, \theta, \phi)$, $s=\frac{1}{2}, 0 \leqslant \theta \leqslant \pi, 0 \leqslant \phi<2 \pi$ which interact with each other and an external (transverse) field. The classical isotropic $X Y$ chain is described by the Hamiltonian

$$
\begin{aligned}
H & =\sum_{n} \Omega s \cos \theta_{n} \\
& +\sum_{n} J s^{2} \sin \theta_{n} \sin \theta_{n+1} \cos \left(\phi_{n}-\phi_{n+1}\right)
\end{aligned}
$$

(compare with (1)). The Hamiltonian of the classical transverse Ising chain contains $\cos \phi_{n} \cos \phi_{n+1}$ instead of $\cos \left(\phi_{n}-\phi_{n+1}\right)$ in the interspin interaction terms in (15). Consider further a chain of period 2 with $\Omega_{1,2}=\Omega \pm \Delta \Omega$, $\Delta \Omega \geqslant 0, J_{1,2}=J$. The ground-state energy ansatz for both the isotropic $X Y$ and transverse Ising chains reads

$$
\begin{aligned}
& E_{0}\left(\theta_{1}, \theta_{2}\right)=\frac{N}{2} s\left((\Omega+\Delta \Omega) \cos \theta_{1}\right. \\
& \left.\quad+(\Omega-\Delta \Omega) \cos \theta_{2}\right)-N|J| s^{2} \sin \theta_{1} \sin \theta_{2}
\end{aligned}
$$

and $\theta_{1}, \theta_{2}$ are determined from the equations

$$
\begin{aligned}
& (\Omega+\Delta \Omega) \sin \theta_{1}+2|J| s \cos \theta_{1} \sin \theta_{2}=0 \\
& (\Omega-\Delta \Omega) \sin \theta_{2}+2|J| s \sin \theta_{1} \cos \theta_{2}=0
\end{aligned}
$$

to provide a minimum of $E_{0}\left(\theta_{1}, \theta_{2}\right)(16)$. The on-site magnetizations have the component arbitrary directed in the $x y$ plane with $\left|m_{\perp n}\right|=$ $s \sin \theta_{n}(n=1,2)$ for the isotropic $X Y$ case or the component directed along the $x$ axis with $\left|m_{x n}\right|=s \sin \theta_{n}$ for the transverse Ising case and the $z$ component given by $m_{n}^{z}=s \cos \theta_{n}$ in both cases. The components of the total magnetization per site are then as follows $m_{\perp}=$

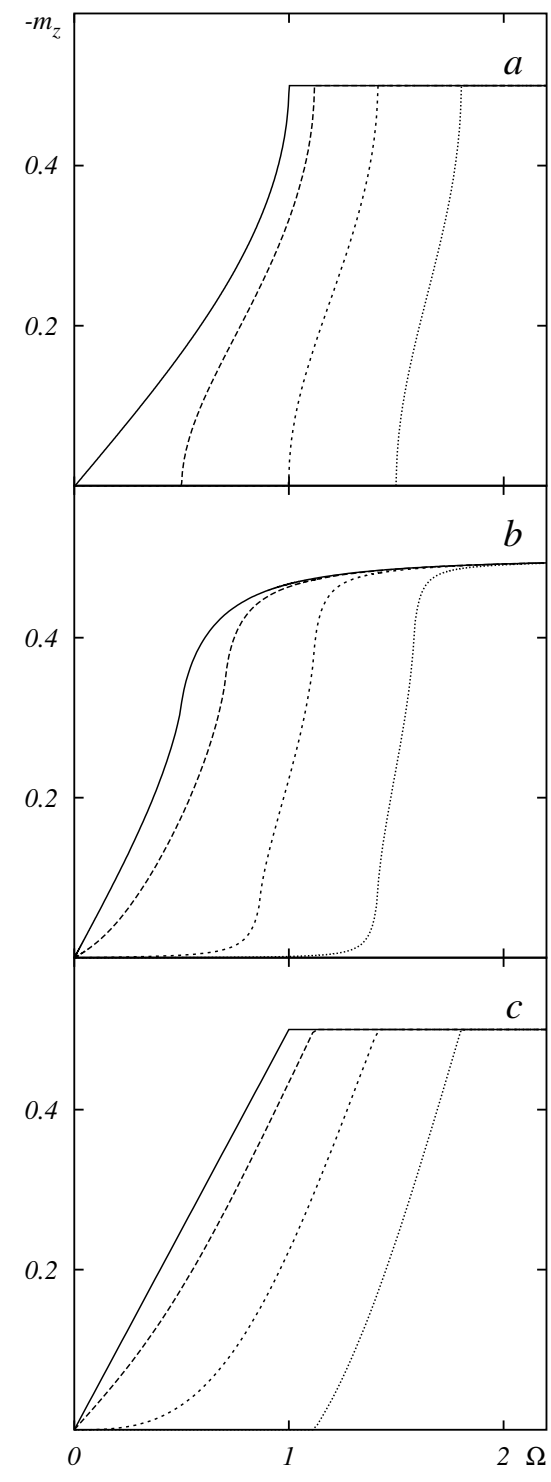

Figure 3. The zero-temperature magnetization curves for the isotropic $X Y$ (a), transverse Ising (b) and classical (c) chains of period $2\left(J_{n}=1, \Omega_{1,2}=\right.$ $\Omega \pm \Delta \Omega, \Delta \Omega=0$ (solid curves), $\Delta \Omega=0.5$ (long-dashed curves), $\Delta \Omega=1$ (short-dashed curves), $\Delta \Omega=1.5$ (dotted curves)).

\footnotetext{
${ }^{5}$ Rigorous calculations of the initial static susceptibility tensor for another nonuniform classical chain, i.e., the spin- $\frac{1}{2}$ Ising chain, can be found in [38].
} 
$\frac{1}{2}\left(m_{\perp 1}+m_{\perp 2}\right)$ or $m_{x}=\frac{1}{2}\left(m_{x 1}+m_{x 2}\right)$ (if $\left.J<0\right)$ and $m_{z}=\frac{1}{2}\left(m_{z 1}+m_{z 2}\right)$. Equations (17) may be solved analytically yielding the ground-state energy and the onsite magnetizations $m_{\perp 1}\left(m_{x 1}\right), m_{\perp 2}\left(m_{x 2}\right), m_{z 1}, m_{z 2}$. In figure 3 we contrast the zero-temperature magnetization curves for the isotropic $X Y$, transverse Ising and classical chains of period 2. Note, that the classical isotropic $X Y$ chain similarly to its quantum counterpart may exhibit plateaus in the dependence $m_{z}$ vs. $\Omega$. Evidently, it would be interesting to examine a quantum-to-classical crossover in the magnetization curves. Probably a real-space renormalization-group method aimed on the study of quantum fields on a lattice and applied in [39] to the spin- $\frac{1}{2}$ transverse Ising chain may be used to analyse the spin-1 chain, that is, an intermediate case between the quantum $\left(s=\frac{1}{2}\right)$ and the classical $(s \rightarrow \infty)$ cases.

Exact results for the magnetization curves of spin- $\frac{1}{2} X Y$ chains may be employed to test the strong-coupling approach that is a widely used approximate approach in the theory of low-dimensional spin systems consisting of periodically repeating units (spin chains with a periodic modulation of the intersite interactions or spin ladders) [40].

\subsection{Spin-Peierls instability}

Since the paper of P.Pincus [41] we know that the spin- $\frac{1}{2}$ isotropic $X Y$ chain is unstable with respect to a lattice dimerization due to the spin-Peierls mechanism ${ }^{6}$. Really, the ground-state energy per site of the dimerized spin chain (i.e., with $J_{n}=$ $\left.J\left(1-(-1)^{n} \delta\right)\right)$ is given by

$$
\begin{array}{r}
e_{0}(\delta)=-\frac{|J|}{\pi} \int_{0}^{\psi} \mathrm{d} \varphi \sqrt{1-\left(1-\delta^{2}\right) \sin ^{2} \varphi}-|\Omega|\left(\frac{1}{2}-\frac{\psi}{\pi}\right), \\
\psi=\left\{\begin{array}{lll}
0, & \text { if } & |J| \leqslant|\Omega|, \\
\arcsin \sqrt{\frac{1-\Omega^{2} / J^{2}}{1-\delta^{2}}}, & \text { if } & \delta|J| \leqslant|\Omega|<|J|, \\
\frac{\pi}{2}, & \text { if } & |\Omega|<\delta|J| .
\end{array}\right.
\end{array}
$$

At $\Omega=0$ the ground-state energy per site $(18)\left(e_{0}(\delta) \approx e_{0}(0)+\frac{|J|}{2 \pi} \delta^{2} \ln \delta, \delta \ll 1\right)$ decreases rapidly enough in comparison with the increase of the elastic energy per site $\alpha \delta^{2}$ as $\delta$ increases to provide a minimum of the total energy per site $e_{0}(\delta)+\alpha \delta^{2}$ at a nonzero value of the dimerization parameter $\delta^{\star}$. The external field may destroy dimerization: if $\Omega$ exceeds a certain value the dimerized phase cannot survive and the uniform phase becomes favourable. The phase diagram shown in figure 4a specifies the region of stability/metastability of the dimerized phase at zero temperature.

It is generally known (see, for example, a review on $\mathrm{CuGeO}_{3}$ [43]) that the increase of external field leads to a transition from the dimerized phase to the incommensurate phase rather than to the uniform phase that contradicts to what is seen in figure 4. Obviously, the incommensurate phase cannot appear within the frames of the adopted ansatz for lattice distortion $\delta_{1} \delta_{2} \delta_{1} \delta_{2} \ldots, \delta_{1}+\delta_{2}=0$. Therefore, we

\footnotetext{
${ }^{6}$ This conclusion was obtained within the adiabatic approximation; the corresponding treatment within the nonadiabatic approximation is more sophisticated [42].
} 
should assume $J_{n}=J\left(1+\delta_{n}\right)$ and examine the total energy $E_{0}\left(\left\{\delta_{n}\right\}\right)+\sum_{n} \alpha \delta_{n}^{2}$ for different lattice distortions $\left\{\delta_{n}\right\}$. We introduce a trial distortion of the form

$$
\delta_{n}=-\delta \cos \left(\frac{2 \pi}{p} n\right)
$$

where $p$ is the period of modulation and analyse numerically $(N=1000)$ the total energy to reveal which spin-Peierls phase is realized in the presence of external field (for details see [44]). Comparing the behaviour of the total energy for $p=2$ and $p=1.9$, $p=2.1$ as $\Omega$ increases we conclude that a long-period structure does arise if $\Omega$ exceeds a certain value. The dimerized phase transforms into a long-period phase rather than into the uniform phase while the field increases. The important conclusion of further computations using (19) is that the dimerized phase persists up to a certain characteristic field. Further, in moderate fields the lattice parameterized by (19) may exhibit short-period phases, for example, the trimerized phase with $p=3$ [45] (another possible lattice distortion which preserves the chain length is $\delta_{1}=-\delta_{2}$ ). However, a behaviour of the trimerized phase is essentially different in comparison with that of the dimerized phase: for any small deviation of the field from the value at which the trimerized phase occurs there exists such a long-periodic structure for which the lattice distortion (19) gives smaller total energy than for $p=3$. Thus, contrary to the dimerized phase, the trimerized phase does not persist with the field varying and it continuously transforms into a certain long-period phase with the field varying. In strong fields the uniform lattice can be expected. Clearly, such a study is restricted to the adopted ansatz (19) (although any other (but not all) distortion pattern may be assumed) and therefore we can say for sure which lat-

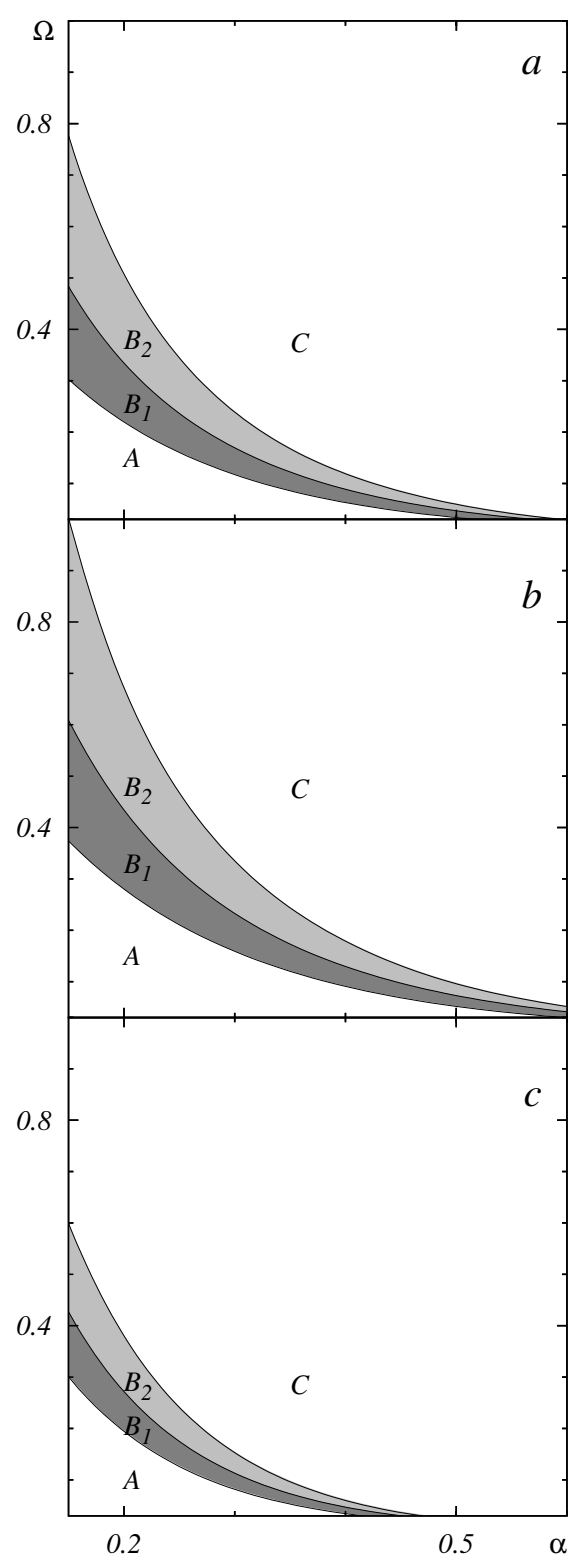

Figure 4. The phase diagram of the isotropic $X Y$ chain $(J=1)$ in the plane lattice stiffness $\alpha$ - transverse field $\Omega$ which indicates the regions of stability/metastability of the dimerized and uniform phases at zero temperature. A (C) - only dimerized (uniform) phase occurs; $\mathrm{B}_{1}\left(\mathrm{~B}_{2}\right)$ both phases are possible but the dimerized (uniform) is the favourable one. The phase diagram also illustrates the possible effects of the DzyaloshinskiiMoriya interaction (a: $D=0$, b: $D=$ $0.5, k=1$, c: $D=0.5, k=0)$. 
tice distortion is not realized rather than to point out which lattice distortion should occur.

It is interesting to discuss the effect of the Dzyaloshinskii-Moriya interaction on the spin-Peierls dimerization. For this purpose we should find the ground-state energy of the chain of period 2 with a sequence of parameters

$$
\Omega J_{1} D_{1} \Omega J_{2} D_{2} \Omega J_{1} D_{1} \Omega J_{2} D_{2} \ldots
$$

Moreover, $J_{1,2}=J(1 \pm \delta)$ and $D_{1,2}=D(1 \pm k \delta)$. Putting $k=0$ one has a chain in which $D$ does not depend on the lattice distortion, whereas for $k=1$ the dependence of $D$ on the lattice distortion is the same as that for the isotropic $X Y$ exchange interaction. In the limit of small $\delta \ll 1$ (valid for lattices with large values of $\alpha$ and corresponding to the experimental situation) the analysis becomes extremely simple. It is convenient to introduce the parameter

$$
\aleph=\frac{J^{2}+k D^{2}}{J^{2}+D^{2}}
$$

Consider, for example, the case $\Omega=0$. After a simple rescaling of variables one arrives at the equations considered in [41] and as a result

$$
\delta^{\star} \sim \frac{1}{\aleph} \exp \left(-\frac{2 \pi \alpha}{\sqrt{J^{2}+D^{2} \aleph^{2}}}\right)
$$

We immediately conclude that for $k=1$ nonzero $D$ leads to the increase of the dimerization parameter $\delta^{\star}$, whereas for $k=0$ nonzero $D$ leads to the decrease of $\delta^{\star}$. Thus, the Dzyaloshinskii-Moriya interaction may act either in favour of the dimerization or against it. The actual result of its effect depends on the dependence of the Dzyaloshinskii-Moriya interaction on the amplitude of the lattice distortion in comparison with the corresponding dependence of the isotropic $X Y$ exchange interaction. Comparing figure $4 \mathrm{a}$ and figures $4 \mathrm{~b}$, $4 \mathrm{c}$ one can see the described effects of the Dzyaloshinskii-Moriya interaction on the spin-Peierls dimerization.

Finally, let us make some remarks on the exchange interaction anisotropy effects on the spin-Peierls dimerization. Consider the anisotropic $X Y$ chain (without field). In the isotropic limit it exhibits the spin-Peierls dimerized phase, whereas in the extremely anisotropic limit (Ising chain) the ground-state energy does not depend on $\delta$ and the spin-Peierls dimerization cannot occur. Therefore, it is attracting to follow how anisotropy of the exchange interaction destroys the dimerized phase and to find, in particular, a critical value of anisotropy above which the dimerized phase cannot appear. The consideration becomes even more complicated in the presence of an external (transverse) field. Note, however, that such a study could be performed many years ago on the basis of the results for the anisotropic $X Y$ chain in a transverse field of period 2 reported in [35].

Another issue which deserves to be studied in detail is related to a spin-Peierls instability of the spin- $\frac{1}{2}$ transverse Ising chain [33]. By direct inspection one can make sure that although the ground-state energy of the dimerized Ising chain in a constant 
transverse field $\Omega$ decreases as $\delta$ increases, however, not sufficiently rapidly (for any $\Omega$ ) to get a gain in the total energy. As a result no spin-Peierls dimerization should be anticipated. On the other hand, the transverse Ising chain is unitary equivalent to the anisotropic $X Y$ chain without field. By comparison of the diagonal Green functions (see equation (14) in the present paper and equation (8) in [33, J. Phys. A]) it can be found that the Helmholtz free energy of the anisotropic $X Y$ chain without field of $N$ sites $I_{n}^{x} I_{n}^{y} I_{n+1}^{x} I_{n+1}^{y} \ldots$ is the sum of the Helmholtz free energies of two transverse Ising chains $\Omega_{n} I_{n} \Omega_{n+1} I_{n+1} \ldots$ of $N / 2$ sites, namely,

$$
\ldots I_{n-1}^{x} I_{n}^{y} I_{n+1}^{x} I_{n+2}^{y} \ldots
$$

and

$$
\ldots I_{n-1}^{y} I_{n}^{x} I_{n+1}^{y} I_{n+2}^{x} \ldots
$$

Consider at first the following isotropic $X Y$ chain $I_{n}^{x}=I_{n}^{y}=I\left(1-(-1)^{n} \delta\right)$ which exhibits the spin-Peierls dimerization. In view of the mentioned correspondence the introduced model is thermodynamically equivalent to two uniform transverse Ising chains (both with the transverse field $I(1+\delta)$ (or $I(1-\delta))$ and the Ising exchange interaction $I(1-\delta)$ (or $I(1+\delta))$ ) and thus we conclude that the critical Ising chain $(\Omega=I)$ is unstable with respect to a uniform extending (or shortening) accompanied by the corresponding increase (decrease) of the transverse field. Further, it is known that the quadrimerized isotropic $X Y$ chain without field may be energetically favourable in comparison with the uniform chain (although yielding a smaller gain in the total energy than the dimerized chain). Consider, therefore, such a chain of period 4 with $I_{n}=I^{\prime}(1+\delta), I_{n+1}=I^{\prime \prime}(1+\delta), I_{n+2}=I^{\prime}(1-\delta), I_{n+3}=I^{\prime \prime}(1-\delta)$ which is unstable with respect to a lattice distortion characterizing by nonzero value of $\delta$ for certain values of $I^{\prime}, I^{\prime \prime}$. This model exhibits the same thermodynamics as two identical transverse Ising chains of period $2 \Omega_{n} I_{n} \Omega_{n+1} I_{n+1} \Omega_{n} I_{n} \Omega_{n+1} I_{n+1} \ldots$ with $\Omega_{n}=I^{\prime \prime}(1-\delta), \Omega_{n+1}=I^{\prime \prime}(1+\delta)$ and $I_{n}=I^{\prime}(1+\delta), I_{n+1}=I^{\prime}(1-\delta)$. These arguments indicate a possibility of the spin-Peierls bond dimerization of the spin- $\frac{1}{2}$ transverse Ising chain accompanied by the coherent modulation of the on-site transverse fields.

\section{Skipped items and summary}

Needless to say that many important contributions have appeared out of the scope of this brief survey. Let us discuss in a telegraph-style manner some of them. Numerous works were devoted to the analysis of the properties of spin- $\frac{1}{2} X Y$ chains with aperiodic or random Hamiltonian parameters [46] (as, for example, an extensive real-space renormalization-group treatment of the random transverse Ising chain by D.S.Fisher).

One of the interesting subjects in the theory of magnetic materials is the magnetic relaxation in spin systems and, in particular, the impurity spin relaxation in spin systems. Spin- $\frac{1}{2} X Y$ chains provide a possibility to rigorously study the relaxation phenomena in one-dimensional spin models containing impurities [47,3]. A single impurity may be assumed in the sense that the interaction with its neighbours is 
different in strength $\left(J^{\prime} \neq J\right)$. The impurity spin may be located either at the boundary of the system $\left(J_{1}=J^{\prime}, J_{2}=J_{3}=\ldots=J\right)$ or in the bulk (e.g., $J_{\frac{N}{2}-1}=$ $J_{\frac{N}{2}}=J^{\prime}$ and $J_{n}=J$ for all other $n$ ). After the Jordan-Wigner transformation one faces a chain of tight-binding fermions with the impurity site (in the sense that the hopping amplitude(s) surrounding this site is (are) different in value) the energy spectrum of which is well-known (if $J^{\prime}<J_{c}$ the elementary excitation energies form a band whereas if $J^{\prime}>J_{c}$ two states emerge from the band; moreover, $J_{c}=\sqrt{2} J$ $\left(J_{c}=J\right)$ for the boundary (bulk) impurity). Nevertheless, the time dependence of the equilibrium autocorrelation functions $\left\langle s_{n}^{\alpha}(t) s_{n}^{\alpha}\right\rangle$ is not obvious since they are two-fermion $(z z)$ or, generally speaking, many-fermion $(x x)$ quantities. The timedependent autocorrelation functions $\left\langle s_{n}^{\alpha}(t) s_{n}^{\alpha}\right\rangle$ for the impurity spin or for the spins in its vicinity may exhibit new types of asymptotic behaviour depending on the relation between $J$ and $J^{\prime}$ and temperature. The impurity relaxation may become even more complicated in the presence of an external (transverse) field.

Spin- $\frac{1}{2} X Y$ chains have been used to study the nonequilibrium properties of quantum systems [48]. Z.Rácz with coworkers suggested to consider a nonequilibrium system imposing a current on a system and investigating the steady states. Alternatively, we can gain an understanding of the nonequlibrium properties examining the dynamics of an initial state (for example, a kink or droplet configuration of $z$ on-site magnetizations).

Recently a study of the transport properties (for example, of the thermal conductivity) of low-dimensional spin systems which are significantly determined by magnetic excitations have attracted much interest [49]. A simple case of the isotropic $X Y$ chain emerges in such studies as a milestone providing reference results for more sophisticated models.

Finally, some exotic applications of spin- $\frac{1}{2} X Y$ chains have appeared recently. For example, in connection with quantum information processing the numerical/analytical computations of entanglement in spin- $\frac{1}{2} X Y$ models on one-dimensional lattices of small/infinite number of sites were carried out [50]. A study of the correlation function which is called the emptiness formation probability (i.e., the probability of the formation of a ferromagnetic string in the antiferromagnetic ground state) yields interesting links between statistical mechanics and number theory. The case of the isotropic $X Y$ chain provides a valuable background for calculation of such correlation functions [51].

We hope that the present review shows that spin- $\frac{1}{2} X Y$ chains still contain quite unexplored properties which deserve to be discussed. A serious advantage of this type of models is a possibility to perform statistical mechanical calculations either rigorously analytically or exactly numerically (considering as long chains as required to obtain results which pertain to the thermodynamic limit). On the other hand, it is always desirable to clarify afterwards the relation of the obtained results to more realistic models (with the Heisenberg interaction, interchain interaction, $s>\frac{1}{2}$ ). Many interesting questions in the theory of these quantum spin chains still remain open and call for new efforts. 


\section{Acknowledgements}

The author thanks Taras Krokhmalskii, Taras Verkholyak and Oles' Zaburannyi for discussions. This work was partly supported by the STCU under the project No. 1673. The author is grateful to doctor Janush Sanotsky who made it possible to prepare the paper in due time.

\section{References}

1. Kenzelmann M., Coldea R., Tennant D.A., Visser D., Hofmann M., Smeibidl P., Tylczynski Z. // Phys. Rev. B, 2002, vol. 65, 144432.

2. Farias G.A., Gonçalves L.L. // Physica status solidi (b), 1987, vol. 139, p. 315.

3. Stolze J., Nöppert A., Müller G. // Phys. Rev. B, 1995, vol. 52, p. 4319; Stolze J., Vogel M. // Phys. Rev. B, 2000, vol. 61, p. 4026.

4. Asakawa H. // Physica A, 1996, vol. 233, p. 39.

5. Young A.P., Rieger H. // Phys. Rev. B, 1996, vol. 53, p. 8486; Young A.P. // Phys. Rev. B, 1997, vol. 56, p. 11691; Sachdev S., Young A.P. // Phys. Rev. Lett., 1997, vol. 78 , p. 2220.

6. Derzhko O., Krokhmalskii T. // Fizika Nizkikh Temperatur (Kharkiv), 1997, vol. 23, p. 721; Phys. Rev. B, 1997, vol. 56, p. 11659; Physica status solidi (b), 1998, vol. 208, p. 221.

7. Braeter H., Kowalski J.M. // Physica A, 1977, vol. 87, p. 243; Derzhko O., Krokhmalskii T. // Visnyk L'viv. univ., ser. fiz., 1993, No. 26, p. 47 (in Ukrainian); Ferroelectrics, 1994, vol. 153, p. 55; J. Magn. Magn. Mater., 1995, vol. 140-144, p. 1623; Visnyk L'viv. univ., ser. fiz., 1995, No. 27, p. 21 (in Ukrainian); Ferroelectrics, 1997, vol. 192, p. 21; Derzhko O., Krokhmalskii T., Verkholyak T. // J. Magn. Magn. Mater., 1996, vol. 157/158, p. 421; Materials Science \& Engineering A, 1997, vol. 226-228, p. 1049; Philosophical Magazine B, 1997, vol. 76, p. 855.

8. Taylor J.H., Müller G. // Physica A, 1985, vol. 130, p. 1; Viswanath V.S., Müller G. The Recursion Method. Application to Many-body Dynamics. Berlin, Heidelberg, Springer-Verlag, 1994.

9. Derzhko O., Krokhmalskii T., Stolze J. // J. Phys. A, 2000, vol. 33, p. 3063; Czechoslovak Journal of Physics, 2002, vol. 52, p. 321; J. Phys. A, 2002, vol. 35, p. 3573.

10. Derzhko O., Krokhmalskii T. // Physica status solidi (b), 2000, vol. 217, p. 927; Annalen der Physik (Leipzig), 1999, vol. 8, p. SI-45.

11. Barnes T. Preprint cond-mat/0204115.

12. Perk J.H.H., Capel H.W. // Physica A, 1980, vol. 100, p. 1.

13. Rao S., Sen D. Preprint cond-mat/0005492 (and references therein).

14. de Lima J.P., Gonçalves L.L. // Physica A, 2002, vol. 311, p. 458.

15. Plascak J.A., Pires A.S.T., Sá Barreto F.C. // Solid State Commun., 1982, vol. 44, p. 787; Watarai S., Matsubara T. // J. Phys. Soc. Jpn., 1984, vol. 53, p. 3648; Derzhko O.V., Levitskii R.R., Sorokov S.I. // Ukrainian Journal of Physics, 1990, vol. 35, p. 1421 (in Ukrainian); Florencio J., Sá Barreto F.C. // Phys. Rev. B, 1999, vol. 60 , p. 9555.

16. Yukhnovskii I.R., Levitskii R.R., Sorokov S.I., Derzhko O.V. // Izv. AN SSSR, ser. fiz., 1991, vol. 55, p. 481 (in Russian); Sorokov S.I., Levitskii R.R. Thermodynamics and longitudinal dynamical properties of the 1D Ising model in a transverse field. 
Preprint of the Institute for Condensed Matter Physics, ICMP-94-3E, L'viv, 1994, 20 p.; Levitskii R.R., Sokolovskii R.O., Sorokov S.I. // Condens. Matter Phys., 1997, No. 10, p. 67; Levitskii R.R., Sorokov S.I., Baran O.R. // Condens. Matter Phys., 2000, vol. 3, p. 515 .

17. Derzhko O. // Journal of Physical Studies (L'viv), 2001, vol. 5, p. 49; Derzhko O., Krokhmalskii T. // Acta Physica Polonica B, 2001, vol. 32, p. 3421; J. Magn. Magn. Mater., 2002, vol. 242-245, p. 778; Derzhko O., Richter J., Verkholyak T. // Acta Physica Polonica B, 2001, vol. 32, p. 3427; Czechoslovak Journal of Physics, 2002, vol. 52, p. A41; Derzhko O., Verkholyak T., Schmidt R., Richter J. Preprint cond-mat/0207179 (to appear in Physica A); Nunner T.S., Kopp T. Preprint cond-mat/0210103.

18. Schulz H.J. // Phys. Rev. B, 1986, vol. 34, p. 6372; Kolezhuk A.K., Mikeska H.-J. Preprint cond-mat/0202171.

19. Tsukada I., Takeya J., Masuda T., Uchinokura K. // Phys. Rev. Lett., 2001, vol. 87, 127203.

20. Kontorovich V.M., Tsukernik V.M. // Zh. Eksp. Teor. Fiz., 1967, vol. 52, p. 1446 (in Russian); Siskens Th., Capel H.W., Gaemers K.J.F. // Physica A, 1975, vol. 79, p. 259; Siskens Th., Capel H.W. // Physica A, 1975, vol. 79, p. 296; Zvyagin A.A. // Phys. Lett. A, 1991, vol. 158, p. 333; Daniel M., Amuda R. // Phys. Rev. B, 1996, vol. 53, p. R2930; Gottlieb D., Rössler J. // Phys. Rev. B, 1999, vol. 60, p. 9232; Pires A.S.T. // J. Magn. Magn. Mater., 2001, vol. 223, p. 304.

21. Oshikawa M., Affleck I. // Phys. Rev. Lett., 1997, vol. 79, p. 2883; Aristov D.N., Maleyev S.V. // Phys. Rev. B, 2000, vol. 62, p. R751; Derzhko O., Richter J., Zaburannyi O. // J. Phys.: Condens. Matter, 2000, vol. 12, p. 8661.

22. Derzhko O.V., Levitskii R.R., Moina A.Ph. // Condens. Matter Phys., 1993, No. 1, p. 115 (in Ukrainian); Derzhko O.V., Moina A.Ph. // Condens. Matter Phys., 1994, No. 3, p. 3; Ferroelectrics, 1994, vol. 153, p. 49; Physica status solidi (b), 1996, vol. 196, p. 237.

23. Bocquet M., Essler F.H.L., Tsvelik A.M., Gogolin A.O. Preprint cond-mat/0102138.

24. Lloyd P. // J. Phys. C, 1969, vol. 2, p. 1717; Nishimori H. // Phys. Lett. A, 1984, vol. 100, p. 239; Derzhko O., Verkholyak T. // Physica status solidi (b), 1997, vol. 200, p. 255; Materials Science \& Engineering A, 1997, vol. 226-228, p. 745; Fizika Nizkikh Temperatur (Kharkiv), 1997, vol. 23, p. 977.

25. John W., Schreiber J. // Physica status solidi (b), 1974, vol. 66, p. 193; Richter J., Handrich K., Schreiber J. // Physica status solidi (b), 1975, vol. 68, p. K61; Richter J., Schreiber J., Handrich K. // Physica status solidi (b), 1976, vol. 74, p. K125; Richter J. // Physica status solidi (b), 1978, vol. 87, p. K89.

26. Derzhko O., Richter J. // Phys. Lett. A, 1996, vol. 222, p. 348; Phys. Rev. B, 1997, vol. 55, p. 14298; Phys. Rev. B, 1999, vol. 59, p. 100.

27. Derzhko O., Richter J., Derzhko V. // Annalen der Physik (Leipzig), 1999, vol. 8, p. SI-49.

28. Gonçalves L.L., Vieira A.P. // J. Magn. Magn. Mater., 1998, vol. 177/181, p. 79.

29. Derzhko O., Krokhmalskii T. // Journal of Physical Studies (L'viv), 1998, vol. 2, p. 263; Derzhko O., Krokhmalskii T., Zaburannyi O. // Condens. Matter Phys., 1999, vol. 2 , p. 339 .

30. Nieuwenhuizen T.M. // J. Phys. A, 1984, vol. 17, p. 1111; Nieuwenhuizen T.M., Luck J.M. // J. Phys. A, 1986, vol. 19, p. 1207; Luck J.M., Nieuwenhuizen T.M. // J. Phys. A, 1989, vol. 22, p. 2151; Funke M., Nieuwenhuizen T.M., Trimper S. 
// J. Phys. A, 1989, vol. 22, p. 5097; Luck J.M., Funke M., Nieuwenhuizen T.M. // J. Phys. A, 1991, vol. 24, p. 4155.

31. de Lima J.P., Gonçalves L.L. // J. Magn. Magn. Mater., 1995, vol. 140-144, p. 1606; J. Magn. Magn. Mater., 1999, vol. 206, p. 135; Barbosa Filho F.F., de Lima J.P., Gonçalves L.L. // J. Magn. Magn. Mater., 2001, vol. 226-230, p. 638; Tong P., Zhong M. // Physica B, 2001, vol. 304, p. 91; Strečka J., Jaščur M. // Czechoslovak Journal of Physics, 2002, vol. 52, p. A37.

32. Derzhko O. // Fizika Nizkikh Temperatur (Kharkiv), 1999, vol. 25, p. 575; Derzhko O., Richter J., Zaburannyi O. // Phys. Lett. A, 1999, vol. 262, p. 217; Acta Physica Polonica A, 2000, vol. 97, p. 931; Physica A, 2000, vol. 282, p. 495; J. Magn. Magn. Mater., 2000, vol. 222, p. 207.

33. Derzhko O. // J. Phys. A, 2000, vol. 33, p. 8627; Derzhko O., Zaburannyi O. // Ukrainian Journal of Physics, 2002, vol. 47, p. 599 (in Ukrainian); Derzhko O., Richter J., Zaburannyi O. // J. Magn. Magn. Mater., 2002, vol. 242-245, p. 778; Derzhko O., Richter J., Krokhmalskii T., Zaburannyi O. // Phys. Rev. B, 2002, vol. 66, p. 144401.

34. Derzhko O. // Czechoslovak Journal of Physics, 2002, vol. 52, p. A277.

35. Perk J.H.H., Capel H.W., Zuilhof M.J., Siskens Th.J. // Physica A, 1975, vol. 81, p. 319.

36. Oshikawa M., Yamanaka M., Affleck I. // Phys. Rev. Lett., 1997, vol. 78, p. 1984.

37. Sachdev S. Quantum Phase Transitions. New York, Cambridge University Press, 1999.

38. Idogaki T., Rikitoku M., Tucker J.W. // J. Magn. Magn. Mater., 1996, vol. 152, p. 311; Derzhko O., Zaburannyi O. // Journal of Physical Studies (L'viv), 1998, vol. 2, p. 128; Derzhko O., Zaburannyi O., Tucker J.W. // J. Magn. Magn. Mater., 1998, vol. 186, p. 188.

39. Drell S.D., Weinstein M., Yankielowicz S. // Phys. Rev. D, 1977, vol. 16, p. 1769.

40. Cabra D.C., Grynberg M.D., Honecker A., Pujol P. Preprint cond-mat/0010376 (and references therein); Grynberg M.D., Cabra D.C., Arlego M. // Phys. Rev. B, 2001, vol. 64, p. 134419; Derzhko O. // Ukrainian Journal of Physics, 2001, vol. 46, p. 762.

41. Pincus P. // Solid State Commun., 1971, vol. 9, p. 1971; Beni G., Pincus P. // J. Chem. Phys., 1972, vol. 57, p. 3531; Beni G. // J. Chem. Phys., 1973, vol. 58, p. 3200 .

42. Sil S. // J. Phys.: Condens. Matter, 1998, vol. 10, p. 8851.

43. Boucher J.P., Regnault L.P. // J. Phys. I France, 1996, vol. 6, p. 1939.

44. Derzhko O., Krokhmalskii T. // Ferroelectrics, 2001, vol. 250, p. 397.

45. Okamoto K. // Solid State Commun., 1992, vol. 83, p. 1039.

46. Pfeuty P. // Phys. Lett. A, 1979, vol. 72, p. 245; Luck J.M. // Journal of Statistical Physics, 1993, vol. 72, p. 417; Fisher D.S. // Phys. Rev. B, 1995, vol. 51, p. 6411; McKenzie R.H. // Phys. Rev. Lett., 1996, vol. 77, p. 4804; Iglói F., Turban L., Karevski D., Szalma F. // Phys. Rev. B, 1997, vol. 56, p. 11031; Henelius P., Girvin S.M. // Phys. Rev. B, 1998, vol. 57, p. 11457; Hermisson J., Grimm U., Baake M. Preprint cond-mat/9706106; Hermisson J. Preprint cond-mat/9808238.

47. Tjon J.A. // Phys. Rev. B, 1970, vol. 2, p. 2411.

48. Antal T., Rácz Z., Sasvári L. // Phys. Rev. Lett., 1997, vol. 78, p. 167; Antal T., Rácz Z., Rákos A., Schütz G.M. // Phys. Rev. E, 1998, vol. 57, p. 5184; Antal T., Rácz Z., Rákos A., Schütz G.M. // Phys. Rev. E, 1999, vol. 59, p. 4912; Rácz Z. // Journal of Statistical Physics, 2000, vol. 101, p. 273; Ogata Y. // Phys. Rev. E, 2002, 
vol. 66, 016135; Karevski D. // Eur. Phys. J. B, 2002, vol. 27, p. 147; Berim G.O., Cabrera G.G. // Physica A, 1997, vol. 238, p. 211; Berim G.O., Berim S., Cabrera G.G. // Phys. Rev. B, 2002, vol. 66, p. 094401.

49. Heidrich-Meisner F., Honecker A., Cabra D.C., Brenig W. // Phys. Rev. B, 2002, vol. 66, p. $140406(\mathrm{R})$.

50. Fu H., Solomon A.I., Wang X. // J. Phys. A, 2002, vol. 35, p. 4293; Osborne T.J., Nielsen M.A. Preprint quant-ph/0202162; Bose I., Chattopadhyay E. Preprint condmat/0208011.

51. Shiroishi M., Takahashi M., Nishiyama Y. Preprint cond-mat/0106062; Boos H.E., Korepin V.E., Nishiyama Y., Shiroishi M. Preprint cond-mat/0202346; Abanov A.G., Korepin V.E. Preprint cond-mat/0206353.

\title{
$€$ ще порох у порохівницях, або що ще ми можемо дізнатися, студіюючи спін- $\frac{1}{2} X Y$ ланцюжки
}

\author{
О.Держко ${ }^{1,2}$
}

1 Інститут фізики конденсованих систем НАН України, 79011 Львів, вул. Свєнціцького, 1

2 Кафедра теоретичної фізики

Львівського національного університету ім. Івана Франка 79005 Львів, Драгоманова, 12

Отримано 2 вересня 2002 р.

Зроблено огляд деяких недавніх результатів з статистичної механіки одновимірних спін- $\frac{1}{2} X Y$ систем. Особлива увага звернута на динамічні і термодинамічні властивості моделей з взаємодією Дзялошинського-Морія, скорельованим безладом і регулярно змінними параметрами гамільтоніана.

Ключові слова: спін- $\frac{1}{2} X Y$ ланцюжки, взаємодія Дзялошинського-Морія, скорельований безлад, плато намагніченості, спін-Пайєрлсова нестійкість

PACS: $75.10 .-b$ 
